\title{
Relations entre différents types de volumes en peuplements de hêtres (Fagus sylvatica L.)
}

\author{
J. RONDEUX ' "), H. LECOMTE ' 2 ) et A. TOUSSAINT ' 2 ) \\ Faculte des Sciences Agronomiques, Gembloux (Belgique) \\ Technologie Forestière
}

\begin{abstract}
Résumé
Récemment nous avons élaboré (Toussaint et al., 1983) un tarif de cubage peuplement pour les massifs de hêtre (Fagus sylvatica L.) situés dans la partie méridionale de la Belgique. Ce tarif fournit le volume du bois fort de la tige, ramené à l'hectare, en fonction de la surface terrière à l'hectare et de la hauteur dominante. Nous avons élargi les possibilités d'utilisation de ce tarif en lui associant des relations entre, d'une part, une série de rapports entre divers types de volumes et le volume bois fort tige et, d'autre part, la circonférence de l'arbre de surface terrière moyenne du peuplement.
\end{abstract}

Mots clés : Hêtre, tarif de cubage peuplement, assortiments, inventaires forestiers.

\section{Introduction}

Dans une étude récente portant sur l'estimation rapide du volume de peuplements de hêtres principalement situés dans la partie méridionale de la Belgique, nous avons envisagé la construction d'un tarif de cubage «peuplement» (Toussaint et al., 1983). Ce tarif exprime le volume du bois fort de la tige, ramené à l'hectare, en fonction de la surface terrière à l'hectare et de la hauteur dominante des peuplements.

Si pour les essences feuillues ce volume conventionnel est couramment préconisé en matière d’aménagement et utilisé dans la recherche forestière, force est d'admettre qu'il n'a pas trouvé le même écho dans le domaine de l'exploitation et de la commercialisation des produits ligneux.

Nous avons tenté d'élargir les possibilités d'utilisation du tarif conçu à l'échelle des peuplements en lui associant un ensemble de relations permettant d'estimer les volumes relatifs à diverses découpes répondant mieux aux habitudes du commerce et de la mobilisation des bois.

(1) Technologie Forestière et Centre de Recherches et de Promotion Forestières (I.R.S.I.A.).

(2) Convention de recherche «Inventaire des ressources forestières wallonnes» financéc par la Région wallonne. 


\section{Matériel d'étude}

Les données utilisées dans cette étude ont été sélectionnées parmi 600 unités d'échantillonnage de 10 ares installées pour les besoins d'un inventaire portant sur l'ensemble de la forêt wallonne et intéressant, entre autres, 30000 hectares de futaies de hêtres. A certains endroits, les peuplements concernés ont le plus souvent une allure irrégulière et sont constitués, soit de groupes distincts d'arbres, soit de sujets mélangés de dimensions très différentes. Ailleurs, on se trouve en présence d'ensembles relevant globalement de la futaie équienne et parsemés de groupes plus ou moins bien individualisés de quelques ares constituant des mini-futaies équiennes (RoISIN, 1981).

La présente étude a été réalisée sur la base de 385 unités d'échantillonnage dans lesquelles le hêtre intervient pour au moins 66 p. 100 de la surface terrière totale des essences présentes.

\section{Détermination des volumes à l'hectare}

En ce qui concerne l'utilisation de la matière ligneuse, il est habituel de raisonner en termes de volumes de bois d'œuvre ou de volumes commercialisables, même si le volume du bois fort de la tige, très proche du bois total de tige, serait, selon nous, une mesure beaucoup plus pertinente dans les transactions commerciales.

Cependant, pour des raisons liées à la pratique ou aux conventions toujours en vigucur, nous avons déterminé plusieurs types de volumes jusqu'à des découpes strictement limitées en circonférence.

\subsection{Calcul des différents types de volume}

Pour chaque unité inventoriće ( placette»), nous avons déterminé le volume du bois fort de la tige du hêtre au moyen d'une équation de cubage basée sur la circonférence à $1,5 \mathrm{~m}$ du sol et sur la hauteur dominante du peuplement analysé (3). De cette façon, on a pu mettre en cuvre un tarif «peuplement» exprimant le volume du bois fort tige ramené à l'hectare en fonction de la surface terrière à l'hectare et de la hauteur dominante.

L'équation de cubage que nous avons retenue est (Toussaint et al., 1983) :

$$
\mathrm{V}_{2: 2}=2,0312+0,4105 \mathrm{G} \cdot \mathrm{H}_{\mathrm{d}, \mathrm{m}}
$$

si : $\mathrm{V}_{2: 2}=$ volume du bois fort de la tige à l'hectare $\left(\mathrm{en} \mathrm{m}^{3}\right)$ :

$\mathrm{G}=$ surface terrière à l'hectare $\left(\right.$ en $\left.\mathrm{m}^{2}\right)$;

$\mathbf{H}_{\mathrm{t} \text { om }}=$ hauteur dominante (en $\mathrm{m}$ ).

(3) Calculée à partir de la moyenne arithmétique des hauteurs totales des 4 plus gros arbres par unité d’échantillonnage. 
En outre, selon une méthode basée sur l'utilisation de courbes de profil (PaLm, 1982), nous avons déterminé les volumes de tiges jusqu'à 10 découpes différentes (allant de 60 à $130 \mathrm{~cm}$ de circonférence) et jusqu'à la découpe située à $50 \mathrm{p} .100$ de la circonférence à $1,5 \mathrm{~m}$ du sol (Rondeux, 1975, 1976).

Les rapports entre ces volumes «partiels » et le volume du bois fort de la tige ont ensuite été calculés pour chacune des unités d'échantillonnage. Par convention, les calculs ont exclusivement concerné les arbres dont la circonférence à $1,5 \mathrm{~m}$ était au moins égale à la circonférence de la découpe considérée.

\subsection{Relations entre les volumes}

Nous avons tenté de voir s'il était possible d'établir une relation entre les rapports de volume calculés pour toutes les unités d'échantillonnage analysées et une caractéristique propre aux peuplements. Il s'est avéré que la circonférence de l'arbre de surface terrière moyenne, très proche de la circonférence moyenne arithmétique, expliquait de manière très satisfaisante les différents rapports entre volumes.

Compte tenu de l'allure graphique générale des relations, nous leur avons ajusté une équation de la forme suivante (ALDER, 1980):

$$
\mathrm{R}=\frac{\mathrm{V}_{\mathrm{i}}}{\mathrm{V}_{\mathrm{2} z}}=1-\mathrm{a}_{\mathrm{o}} \mathrm{e}^{\left(\mathrm{a}_{1} \mathrm{c}_{\mathrm{k}}\right),}
$$

si : $\mathrm{V}_{\mathrm{i}}=$ volume (en $\mathrm{m}^{3}$, ramené à l'hectare), jusqu'à la découpe $\mathrm{i}$ (en $\mathrm{cm}$ de circonférence) ;

$\mathrm{V}_{22}=$ volume du bois fort de la tige (en $\mathrm{m}^{3}$, ramené à l'hectare) ;

$c_{\mathrm{g}}=$ circonférence de l'arbre de surface terrière moyenne $(\mathrm{en} \mathrm{cm})$;

$\mathbf{a}_{\mathbf{o}}, \mathbf{a}_{1}=$ constantes à déterminer.

Pour des raisons de facilité, cette courbe a ćté ajustée par régression linćaire simple après transformation logarithmique, soit :

$$
\ln (1-R)=a_{0}^{\prime}+a_{1}^{\prime} c_{g}
$$

L'estimation par cette voie du rapport correspondant à une découpe située à 50 p. 100 de la circonférence à $1,5 \mathrm{~m}$ s'est avérée insatisfaisante suite à une trop grande variabilité de peuplement à peuplement. Pour les autres découpes, l'ajustement proposé a donné lieu aux coefficients repris dans le tableau 1.

Nous avons constaté que les coefficients $a^{\prime}{ }_{0}$ et $a^{\prime}{ }_{1}$ étaient bien corrélés avec les circonférences à la découpe $c_{i}$ et qu'il était possible de les exprimer de manière satisfaisante au moyen des équations suivantes :

$$
\begin{gathered}
\mathrm{a}_{0}^{\prime}=-4,21578+0,10419 \mathrm{c}_{\mathrm{i}}-0,00048443 \mathrm{c}_{\mathrm{i}}^{2} \\
{\left[\mathrm{R}^{2}=0,9911\right]} \\
{[\mathrm{CVR}=10,9 \mathrm{p} \cdot 100]} \\
\mathrm{a}_{1}^{\prime}=-0,019378-0,00015667 \mathrm{c}_{\mathrm{i}}+0,0000015163 \mathrm{c}^{2}{ }_{i} \\
{\left[\mathrm{R}^{2}=0,9982\right]} \\
{[\mathrm{CVR}=0,8 \text { p. } 100]}
\end{gathered}
$$




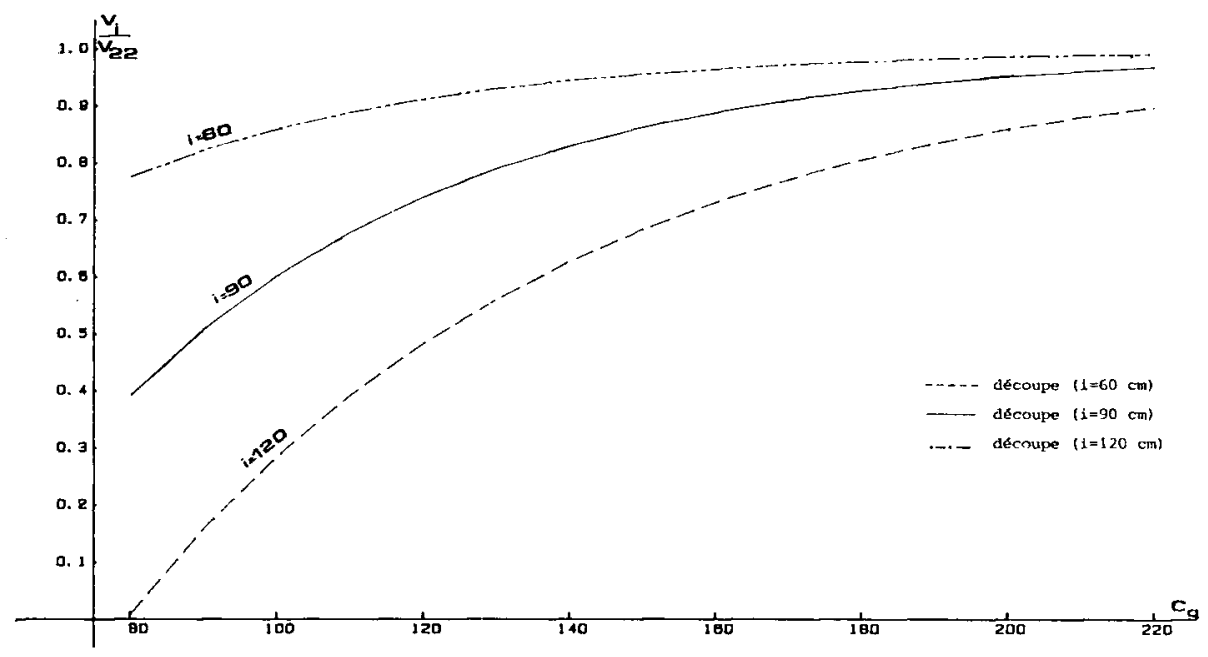

FIi. 1

Relations entre les rapports des volumes correspondant à diverses découpes et le volume bois fort tige $\left(V_{i} / V_{E z}\right)$ et la circonférence de l'arbre de surface terrière moyenne $\left(c_{y}\right)$.

Relations between ratios of volumes $\left(V_{i} / V_{z z}\right)$ and the quadratic mean girth of the stand $\left(c_{, j}\right)$.

\section{TABleav 1}

Valeurs des coefficients $a^{\prime}$ "et $a^{\prime}$, pour les différentes découpes envisagées. Constants $a^{\prime}$, and $a_{1}$ of the equations for the various top girths.

Si l'on accepte une légère perte de précision par rapport aux ajustements individuels, on peut généraliser le modèle initial en remplaçant les coefficients $a_{0}^{\prime}$ et $a_{1}^{\prime}$ dans l'équation de base pour obtenir la nouvelle équation ci-après :

$$
\begin{aligned}
& \ln (1-\mathrm{R})=-4,21578+0,10419 \mathrm{c}_{\mathrm{i}}-0,00048443 \mathrm{c}^{2}{ }_{\mathrm{i}} \\
& -0,019378 \mathrm{c}_{r}-0,00015667 \mathrm{c}_{\mathrm{i}} \mathrm{c}_{r}+0,0000015163 \mathrm{c}_{\mathrm{r}}{ }_{\mathrm{i}} \mathrm{c}_{\mathrm{g}}
\end{aligned}
$$




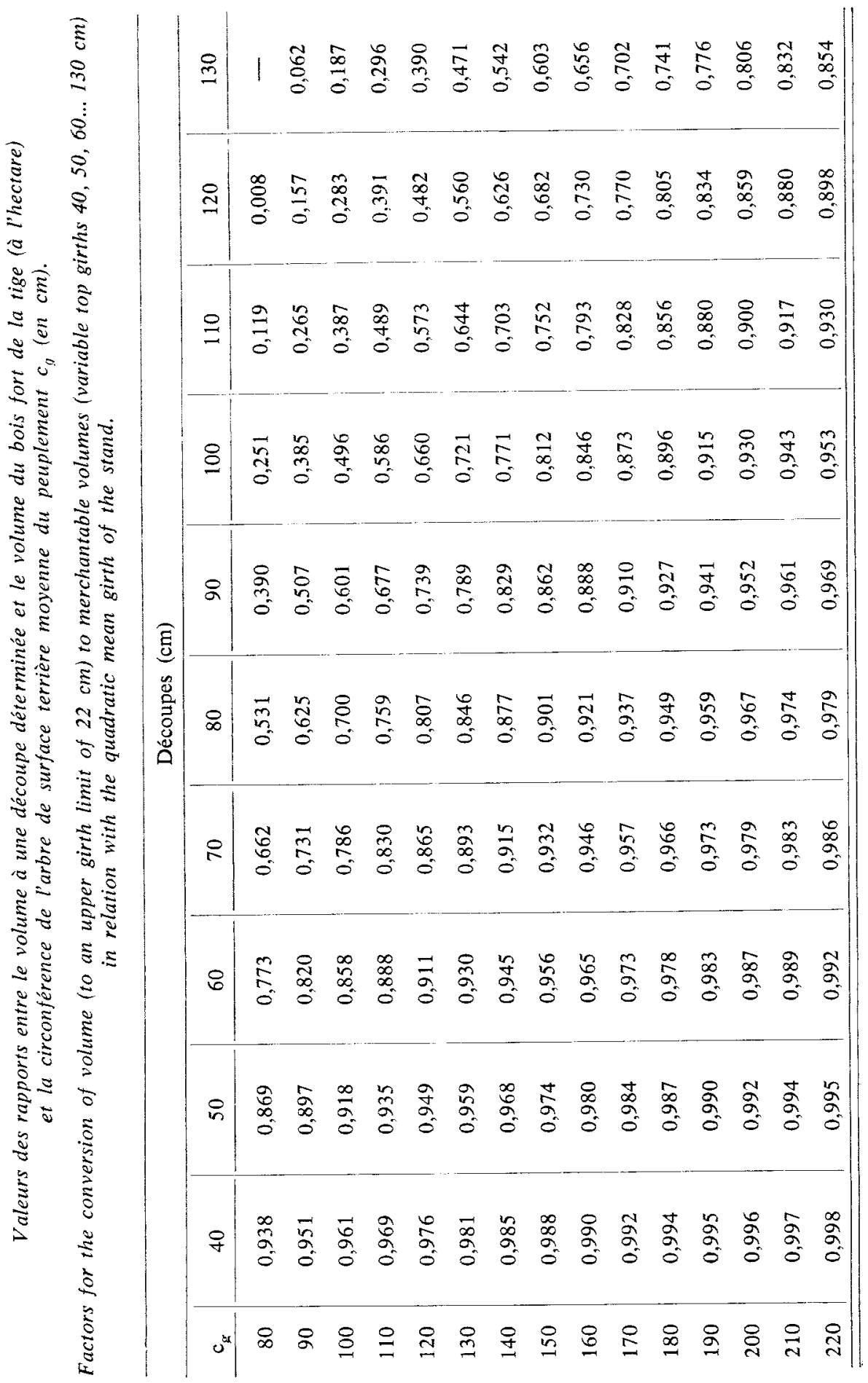




\section{Résultats obtenus et utilisation}

Le tableau 2 fournit les rapports de volumes attendus, obtenus à partir d'ajustements individuels (tabl. 1), pour les découpes envisagées et des circonférences moyennes de peuplements allant de 80 à $220 \mathrm{~cm}$, par classe de 10 en $10 \mathrm{~cm}$. Pour des raisons pratiques, nous avons également matérialisé l'évolution des rapports relatifs aux découpes de $60,90 \mathrm{et} 120 \mathrm{~cm}$ sous forme d'abaques dans la figure 1 .

L'utilisation de ces types de tarif's implique l'estimation de la surface terrière à l'hectare et de la hauteur dominante à partir de plusieurs points répartis de manière alćatoire ou systématique au sein des peuplements ou des massifs à inventorier. La précision obtenue sera donc non seulement fonction de la qualité des estimations de surface terrière et de hauteur dominante, mais aussi du nombre d'échantillons sur lequel s'appuieront ces 2 estimations.

Dans le cas de l'estimation du volume jusqu'à une découpe de $90 \mathrm{~cm}$ de circonférence, par exemple, on pourra écrire que :

ou encore :

$$
\ln (1-R)=1,2034-0,02122 \mathrm{c}_{\mathrm{r}}
$$

$$
\left.\mathrm{R}=\frac{\mathrm{V}_{(0)}}{\mathrm{V}_{\ldots, \ldots}}=1-\mathrm{e}^{(1.2034-0.02122} c_{\mathrm{x}}\right)
$$

Si la circonférence de l'arbre de surface terrière moyenne $c_{x}$ du peuplement étudié est de $1.30 \mathrm{~cm}$, il viendra donc que :

$$
R=1-e^{1,7 n 2}=0,789=78,9 \text { p. } 100
$$

Par utilisation de la relation plus générale impliquant l'harmonisation des coefficients de régression présentés dans le tableau 1, on aurait obtenu :

$$
\mathrm{R}=1-\mathrm{e}^{-1,518 .}=0,781=78 \text { p. } 100
$$

\section{Discussion}

En ce qui concerne la validité de l'extrapolation des relations à l'ensemble des peuplements de hêtre existants, il faut évidemment tenir compte des caractéristiques de ceux qui ont servi de base à l'étude. Les variétés de structure rencontrées au sein de la zone échantillonnée et la grandeur de celle-ci, soit plusieurs dizaines de milliers d'hectares, sont des éléments qui garantissent un champ d'application relativement large (inventaire forestier national, par exemple) aux relations proposćes.

Nous avons pu vérifié que la prise en compte d’un paramètre de dispersion des circonférences des arbres autour de leur moyenne permettait daffiner ces relations et d'accroitre leur fiabilité dans le cas du cubage de peuplements ou d'ensembles de peuplements déterminés. Cette approche est cependant plus laborieuse sur un plan pratique. 
Pour lixer les idées, les peuplements qui ont servi de référence à l'étude sont caractérisés par des hauteurs dominantes variant de 15 à $42 \mathrm{~m}$ (moyenne $27 \mathrm{~m}$ ), des surfaces terrières à l'hectare allant de 6 à $40 \mathrm{~m}^{2}$ (moyenne $19 \mathrm{~m}^{2}$ ) et des circonférences dominantes échelonnées de 60 à $275 \mathrm{~cm}$ (moyenne $174 \mathrm{~cm}$ ), ces dernières traduisant en quelque sorte le stade d'évolution des hêtraies (Toussaint et al., 1983).

\title{
6. Conclusions
}

L'estimation du volume du bois fort de la tige à l'hectare d’un peuplement de hêtres peut être réalisée très rapidement par l'intermédiaire d'un tarif de cubage basé sur la surface terrière à l'hectare et la hauteur dominante de ce peuplement.

Si l'on considère l'ensemble des peuplements inventoriés, les rapports entre les volumes correspondant à diverses découpes définies en circonférence et le volume du bois fort de la tige sont liés de manière très satisfaisante à la circonférence de l'arbre de surface terrière moyenne des peuplements.

Par l'intermédiaire de ces relations, il est possible de valoriser des inventaires rapides de peuplements en estimant non seulement des volumes «aménagement» mais aussi des proportions de volumes de tige correspondant à des découpes en grosseur voisines de celles pratiquées dans les opérations commerciales.

\author{
Reçu le 29 avril 1985. \\ Accepté le 10 aût 1985.
}

\section{Remerciements}

Nous adressons nos remerciements à toutes les personnes, ingénicurs et techniciens, qui de 1979 à 1983 ont inlassablement récolté les données qui ont permis de réaliser cette étude. Notre gratitude va aussi au personnel de l'Administration des Eaux et Forêts pour sa collaboration.

\section{Summary}

Relations between various types of volume in beech stands (Fagus sylvatica L.)

A stand volumc table which provides the volume to a top girth limit of $22 \mathrm{~cm}$ has been rencently constructed (ToussainT et al., 1983) for stands of beech (Fagus sylvatica L.) located in the southern part of Belgium. The ratios between volumes to various fixed girths and volumes to a top girth limit of $22 \mathrm{~cm}$ have been estimated in relation with the girth of the mean basal area tree of the stands. The proposed method is a useful complement to traditional stand volume tables in order to estimate commercial volumes. 


\section{Références bibliographiques}

Alder D., 1980. Estimation des volumes et accroissement des peuplements forestiers avec référence particulière aux forêts tropicales. Vol. 2. Etude et prévision de la production. Etude F.A.O. : Forêts 22/2, Rome, 229 p.

Palm R., 1982. Estimation du profil des arbres et calcul des assortiments. Bull. Rech. Agron. Gembloux, 17, 251-262.

RoISIN P., 1981. Sylviculture des futaies feuillues jardinées ou d'allure jardinée en Belgique. Rev. For. Fr., 33, n" spécial, 113-128.

Rondeux J., 1975. Pour une définition du volume du bois d'œuvre de nos principales essences forestières feuillues. Rev. For. Fr., 27 (5), 371-376.

Rondeux J., 1976. L'opportunité de recoupes strictement définies dans l'estimation du volume du bois d'ouvre (Application au hêtre). Bull. Rech. Agron. Gembloux, 11 (1-2), 265-274.

Toussaint A., Fagot J., Lecomte H., Rondeux J., 1983. Tarif de cubage peuplement pour le hêtre (Fagus sylvatica L.) en Wallonie. Bull. Rech. Agron. Gembloutx 18 (3). 165-172. 